\title{
Augmented Adaptive Control of a Wind Turbine in the Presence of Structural Modes
}

\author{
Susan A. Frost, Member, IEEE \& AIAA, Mark J. Balas, Fellow, IEEE \& AIAA, and Alan D. Wright, \\ Member, ASME
}

\begin{abstract}
Wind turbines operate in highly turbulent environments resulting in aerodynamic loads that can easily excite turbine structural modes, potentially causing component fatigue and failure. Two key technology drivers for turbine manufacturers are increasing turbine up time and reducing maintenance costs. Since the trend in wind turbine design is towards larger, more flexible turbines with lower frequency structural modes, manufacturers will want to develop methods to operate in the presence of these modes. Accurate models of the dynamic characteristics of new wind turbines are often not available due to the complexity and expense of the modeling task, making wind turbines ideally suited to adaptive control. In this paper, we develop theory for adaptive control with rejection of disturbances in the presence of modes that inhibit the controller. We use this method to design an adaptive collective pitch controller for a high-fidelity simulation of a utility-scale, variable-speed wind turbine operating in Region 3. The objective of the adaptive pitch controller is to regulate generator speed, accommodate wind gusts, and reduce the interference of certain structural modes in feedback. The control objective is accomplished by collectively pitching the turbine blades. The adaptive pitch controller for Region 3 is compared in simulations with a baseline classical Proportional Integrator (PI) collective pitch controller.
\end{abstract}

\section{INTRODUCTION}

$\mathrm{R}$ ated wind speed is the velocity at which maximum power output, or rated power, of a wind turbine is achieved. If a turbine is allowed to operate in an uncontrolled manner, in conditions where the wind speed is above the rated wind speed, the power output would increase in proportion to the cube of the wind speed, resulting in overheating of the generator and the power electronics system. Additionally, high wind speeds result in larger aerodynamic forces on the machine, possibly leading to system fatigue and failure. Hence, power output of a turbine must be held constant for wind speeds at and above the turbine's rated wind speed. This wind turbine operation area is called Region 3 [1].

Turbine power output should be maintained at rated power when operating in Region 3. For variable-speed turbines, a constant torque is applied at the generator, and

Manuscript received September 22, 2009

S. A. Frost is with NASA Ames Research Center, Moffett Field, CA 94035 USA (phone: 650-604-0828; fax: 650-604-6594; e-mail: susan.a.frost@nasa.gov).

M. J. Balas is with the Department of Electrical and Computer Engineering, University of Wyoming, Laramie, WY 82071 USA (email: mbalas@uwyo.edu)

A. D. Wright is with the National Renewable Energy Laboratory, DOE, Golden, CO 80401 USA (e-mail: alan_wright@nrel.gov). the turbine rotational speed is maintained at the desired value through the use of blade pitch. In some machines, the pitch angle of each blade is adjusted identically (collective blade pitch); in others the blade pitch is adjusted independently of the other blades (independent blade pitch). Collective blade pitch control is a well-accepted approach to regulating turbine speed and responding to changes in wind speed [2]. Independent blade pitch control is generally used to compensate for asymmetric wind loads, including vertical wind shear, which we ignore in this study.

Wind turbine control problems can benefit from adaptive control techniques [3]-[4], which are well suited to nonlinear applications that have unknown modeling parameters and poorly known operating conditions. The main nonlinearities in a wind turbine model come from the nonlinear aerodynamic loads on the turbine. Creating an accurate model of the dynamic characteristics of a wind turbine is expensive and extremely difficult, if not impossible. Additionally, wind turbines operate in highly turbulent and unpredictable conditions. These complex aspects of wind turbines make them attractive candidates for the application of adaptive control methods. In this paper, we focus on the direct adaptive control (DAC) approach developed in [5][6]. This approach has been extended to handle adaptive rejection of persistent disturbances [7]-[8].

The literature suggests that direct adaptive control methods have rarely been used on utility-scale horizontal axis wind turbines (HAWTs). It was shown in [9] that a pitch controller designed with Direct Model Reference Adaptive Control (DMRAC) was comparable to a PID pitch controller when regulating turbine speed in a simulation of a rigid, nonlinear model of a HAWT. Adaptive pitch control to optimize power in Region 2 of the Controls Advanced Research Turbine (CART) was demonstrated to be effective in simulations and field tests [10].

The CART is a two-bladed, upwind, active-yaw, variablespeed HAWT located at the National Wind Technology Center (NWTC) in Golden, Colorado. This machine is used as a test bed to study aspects of wind turbine control technology for medium-scale machines [1]. The pitch system on the CART uses electromechanical servos that can pitch the blades up to $\pm 18 \mathrm{deg} / \mathrm{s}$.

In Region 3, the CART uses a conventional variablespeed approach to maintain rated electrical power, which is $600 \mathrm{~kW}$ at a low-speed shaft [LSS] speed of 41.7 RPM and a high-speed shaft [HSS] speed of 1800 RPM. Power electronics are used to command constant torque from the generator and full-span blade pitch controls the turbine speed. 
The CART has been modeled with the Fatigue, Aerodynamics, Structures, and Turbulence Codes (FAST) as a combination of rigid and flexible bodies connected by several degrees of freedom (DOFs). The DOFs can be turned on or off individually for analysis purposes by setting a switch in the input data file. The FAST Code is a comprehensive aeroelastic simulator capable of predicting both the extreme loads and the fatigue loads of two- and three-bladed horizontal axis wind turbines [11]. FAST uses Kane's method to set up equations of motion that are solved by numerical integration. The aerodynamic forces and moments along the turbine blade are calculated in FAST by the AeroDyn subroutine package [12]. Results from FAST simulations of the CART compared favorably with field tests on the actual CART [13].

In this paper, we extend our adaptive control theory to accommodate troublesome modal subsystems of a plant that might inhibit the adaptive controller. We use this theory to design a Region 3 adaptive collective pitch controller for the FAST simulation of the CART.

\section{DiRect AdAPtive CONTROL With ReJECTION OF PERSISTENT DistURBANCES}

The adaptive collective pitch controller makes use of a direct adaptive control approach with adaptive rejection of persistent disturbances [8]. We give important details of this theory here. The plant is assumed to be well modeled by the linear, time-invariant, finite-dimensional system:

$$
\left\{\begin{array}{l}
\dot{x}_{p}=A_{p} x_{p}+B_{p} u_{p}+\Gamma_{p} u_{D} \\
y_{p}=C_{p} x_{p} ; x_{p}(0)=x_{0}
\end{array}\right.
$$

where the plant state, $x_{p}$ is an $\mathrm{N}_{\mathrm{p}}$-dimensional vector, the control input vector, $u_{p}$, is M-dimensional, and the sensor output vector, $y_{p}$, is P-dimensional. The disturbance input vector, $u_{D}$, is $\mathrm{M}_{\mathrm{D}}$-dimensional and will be thought to come from the Disturbance Generator:

$$
\left\{\begin{array}{l}
u_{D}=\Theta z_{D} \\
\dot{z}_{D}=F z_{D} ; z_{D}(0)=z_{0}
\end{array}\right.
$$

where the disturbance state, $z_{D}$, is $\mathrm{N}_{\mathrm{D}}$-dimensional. All matrices in (1)-(2) have the appropriate compatible dimensions. Such descriptions of persistent disturbances were first used in [14] to describe signals of known form but unknown amplitude. Sometimes, it is easier to rewrite (2) in a form that is not a dynamical system:

$$
\left\{\begin{array}{l}
u_{D}=\Theta z_{D} \\
z_{D}=L \phi_{D}
\end{array}\right.
$$

where $\phi_{D}$ is a vector composed of the known basis functions for the solution of $u_{D}=\Theta z_{D}$, i.e., $\phi_{D}$ has the basis functions which make up the known form of the disturbance, and $L$ is a matrix of dimension $\mathrm{N}_{\mathrm{D}}$ by $\operatorname{dim}\left(\phi_{D}\right)$. The method for rejecting persistent disturbances used in this paper requires only the knowledge of the form of the disturbance, the amplitude of the disturbance does not need to be known, i.e. $(L, \Theta)$ can be unknown. In this paper, we will be interested in rejecting step disturbances of unknown amplitude which can be represented in the form of (3) as
$\phi_{D} \equiv 1$, with $(L, \Theta)$ unknown.

In [7], as with much of the control literature, it is assumed that the plant and disturbance generator parameter matrices ( $A, B, C, \Gamma, \Theta, F)$ are known. This knowledge of the plant and its disturbance generator allows the Separation Principle of Linear Control Theory to be invoked to arrive at a StateEstimator based, linear controller which can suppress the persistent disturbances via feedback. In this paper, we will not assume that the plant and disturbance generator parameter matrices $(A, B, C, \Gamma, \Theta)$ are known. Here, we will assume that we know the disturbance generator parameter, $F$, from (2), i.e., the form of the disturbance functions is known. In many cases, knowledge of $F$ is not a severe restriction, since the disturbance function is often of known form but unknown amplitude.

Our control objective will be to cause the output of the plant, $y_{p}$, to asymptotically track zero while accommodating disturbances of the form given by the disturbance generator. We define the output error vector as:

$e_{y} \equiv y_{p}-0$

To achieve the desired control objective, we want $e_{y} \underset{t \rightarrow \infty}{\longrightarrow} 0$.

Consider the plant given by (1) with the disturbance generator given by (3). The control objective for this system will be accomplished by an adaptive control law of the form: $u_{p}=G_{e} e_{y}+G_{D} \phi_{D}$

where $G_{e}$ and $G_{D}$ are matrices of the appropriate compatible dimensions, whose definitions will be given later. In [8], the gain adaptation laws were developed to make asymptotic output regulation possible.

Now we specify the adaptive gain laws, which produce asymptotic tracking:

$\left\{\begin{array}{l}\dot{G}_{e}=-e_{y} e_{y}^{T} h_{11} \\ \dot{G}_{D}=-e_{y} \phi_{D}^{T} h_{22}\end{array}\right.$

where $h_{11}$ and $h_{22}$ are arbitrary, positive definite matrices. The adaptive controller is specified by (6) with the above adaptive gain laws (7). See [8] for the stability analysis of this controller and proof that the adaptive gains, $G_{e}$ and $G_{D}$, remain bounded and asymptotic tracking occurs, i.e., $e_{y} \underset{t \rightarrow \infty}{\longrightarrow} 0$.

\section{AdAPtive Collective Pitch CONTROLler}

In this section, we design an adaptive collective pitch controller to regulate generator speed and reject step disturbances. The rated generator speed for the CART is 1800 RPM. The controller objective is accomplished by collectively pitching the turbine blades. See [15] for more details on the design and simulation results of this controller.

The initial plant we use in the FAST simulation of the CART is a two bladed wind turbine model with turbine aerodynamics and generator degree of freedom enabled. The plant neglects all other dynamics and degrees of freedom. The FAST Codes model the nonlinear aerodynamic loads on the turbine. A state space model of the plant was generated by the FAST Codes, see [11], [16] to obtain the model. The 
plant output is generator speed. The control input is the collective pitch angle command.

A classical PI collective pitch controller (the baseline PI pitch controller) has been implemented and tested in the FAST simulation of the CART. A similar version of the baseline PI pitch controller has been field tested on the CART [11]. The baseline PI pitch controller in the FAST simulator provides a basis for comparison with the adaptive pitch controller. The FAST simulator of the CART measures generator speed and feeds it back to the baseline PI pitch controller, which regulates it to the rated generator speed.

The adaptive collective pitch controller designed for this paper replaces the baseline PI pitch controller in the FAST simulator. The adaptive pitch controller was designed with the direct adaptive control approach described in section II.

It has been demonstrated that the uniform wind disturbance, without shear, across the rotor disk of a turbine can be accurately accounted for when modeled as a step disturbance of unknown amplitude [13], [17]. Hence, to improve controller performance we design the adaptive collective pitch controller to reject step disturbances, in addition to regulating generator speed. The objective is accomplished by collective blade pitch.

The control objective is accomplished by an adaptive control law of the form given in (6) with gains specified in (7). For a step function, the disturbance generator function in the form of (3) is specified by $\phi_{D}=1$. Recall that the amplitude of the disturbance function can be unknown, i.e., $(L, \Theta)$ from (3) need not be known. The adaptive control law that accomplishes the control goals described above is:

$\left\{\begin{array}{l}u_{p}=G_{e} e_{y}+G_{D} \\ \dot{G}_{e}=-e_{y} e_{y}^{T} h_{11} \\ \dot{G}_{D}=-e_{y} \phi_{D} h_{22}=-e_{y} h_{22}\end{array}\right.$

where $h_{11}$ and $h_{22}$ are positive scalars.

The adaptive controller specified by (8) was implemented in Simulink in the FAST simulation of the CART. The adaptive controller gains, $h_{11}$ and $h_{22}$, were tuned to minimize the generator speed error, since we had the goal of regulating generator speed, while keeping the blade pitch rate in a range similar to that of the baseline PI controller. The values of the gains used in the adaptive controller were: $h_{11}=6.5$ and $h_{22}=0.3$.

The FAST simulations were run from time 0 seconds to 90 seconds with an integration step size of 0.006 seconds. The simulation used step wind inflow resulting in Region 3 operation of the turbine, see fig. 1(a). The generator DOF was enabled for the simulation. During the initial tests, the other DOF switches were turned off. The wind turbine had fixed-yaw with no yaw control. Aerodynamic forces were calculated during the runs. The parametric information for the FAST simulator as we configured it is available from [11], [16].

In [15], comparisons were made between the baseline PI pitch controller and the adaptive pitch controller described above. Fig. 1(b) shows the generator speed errors for both controllers with step wind inflow after the transients due to startup have died off. The adaptive controller was shown to perform robustly under parameter variations with smaller tracking errors than the baseline PI controller.

We observed poor generator speed tracking from both controllers when the drivetrain rotational-flexibility mode DOF and the first flapwise blade mode DOF were enabled in the simulation, see fig. 2. This observation led us to investigate the feedback control of the turbine in the presence of certain structural modes. In particular, we focused on the first drivetrain rotational-flexibility, or torsional, mode, which is one of the more destructive, easily excited modes in a wind turbine.

The FAST simulation allows for the measurement of many system parameters. We measured the low-speed shaft torque and observed that there was a $3.47 \mathrm{~Hz}$ disturbance in the measurements. This is consistent with the drivetrain torsional mode that is estimated at $3.5 \mathrm{~Hz}$.

A low-pass filter was designed to filter out the drivetrain torsional mode in the generator speed that is fed back to the controller. The results of incorporating the low-pass filter with the baseline PI controller are shown in fig. 3 . The lowpass filter improves the regulation of the generator speed for the PI controller, but the controller still has difficulties, especially for higher wind speeds. A more rigorous technique to reduce the destabilizing effects of structural modes fed back through the controller will now be examined in this paper.

\section{Residual Mode Filter Augmentation of Adaptive CONTROLLER}

In some cases the plant in (1) does not satisfy the requirements of Almost Strictly Positive Real (ASPR) for the use of the adaptive controller (6)-(7). Instead, there maybe be a modal subsystem that inhibits this property. This section will present new results for our adaptive control theory developed in [7]-[8]. We will modify the adaptive controller with a Residual Mode Filter (RMF) to compensate for the troublesome modal subsystem, or the $\mathrm{Q}$ modes, as was done in [18] for fixed gain non-adaptive controllers. Here we present the theory for adaptive controllers augmented with RMFs. In a previous paper, we examined the RMF with adaptive control, but assumed that there was no leakage of the disturbance into the Q modes [19]. Now we will address the issue of disturbances propagating through these modes.

Let us assume that (1) can be partitioned into the following form:

$$
\left\{\begin{array}{l}
{\left[\begin{array}{c}
\dot{x} \\
\dot{x}_{Q}
\end{array}\right]=\left[\begin{array}{ll}
A & 0 \\
0 & A_{Q}
\end{array}\right]\left[\begin{array}{c}
x \\
x_{Q}
\end{array}\right]+\left[\begin{array}{c}
B \\
B_{Q}
\end{array}\right] u_{p}+\left[\begin{array}{c}
\Gamma \\
\varepsilon \Gamma_{Q}
\end{array}\right] u_{D}} \\
y_{p}=\left[\begin{array}{ll}
C & C_{Q}
\end{array}\right]\left[\begin{array}{c}
x \\
x_{Q}
\end{array}\right] ; \varepsilon \geq 0
\end{array}\right.
$$

where $\varepsilon$ represents the amount of leakage of the disturbance into the $\mathrm{Q}$ modal system. To simplify notation, define $x_{p} \equiv\left[\begin{array}{c}x \\ x_{Q}\end{array}\right] ; A_{p}=\left[\begin{array}{cc}A & 0 \\ 0 & A_{Q}\end{array}\right] ; B_{p}=\left[\begin{array}{c}B \\ B_{Q}\end{array}\right] ; C_{p}=\left[C C_{Q}\right]$ 
$\Gamma_{p}=\left[\begin{array}{ll}\Gamma & \varepsilon \Gamma_{Q}\end{array}\right]^{T} ;$ and the disturbance generator is as given before in (2)-(3), i.e., $\left\{\begin{array}{l}\dot{z}_{D}=F z_{D} \\ u_{D}=\theta z_{D}\end{array}\right.$ or $z_{D}=L \phi_{D}$. The Output Tracking Error and control objective remain as in (4)-(5), i.e. $e_{y} \equiv y_{p} \underset{t \rightarrow \infty}{\longrightarrow} 0$.

However, now we will only assume that the subsystem $(A, B, C)$ is ASPR rather than the full un-partitioned plant $\left(A_{p}, B_{p}, C_{p}\right)$, and that the modal subsystem $\left(A_{Q}, B_{Q}, C_{Q}\right)$ will be known and open-loop stable, i.e. $A_{Q}$ is stable. Also note that this subsystem is directly affected by the disturbance input. Recall that ASPR means $C B>0$ and $P(s)=C(s I-A)^{-1} B$ is minimum phase. So, in summary, the actual plant has an ASPR subsystem and a known modal subsystem that is stable but inhibits the property of ASPR for the full plant. Hence, this modal subsystem must be compensated or filtered away.

We define the Residual Mode Filter (RMF):

$$
\left\{\begin{array}{l}
\dot{\hat{x}}_{Q}=A_{Q} \hat{x}_{Q}+B_{Q} u_{p} \\
\hat{y}_{Q}=C_{Q} \hat{x}_{Q}
\end{array}\right.
$$

And the compensated tracking error:

$\tilde{e}_{y} \equiv y_{p}-\hat{y}_{Q}$

Now we let $e_{Q} \equiv \hat{x}_{Q}-x_{Q}$ and obtain:

$\dot{e}_{Q}=A_{Q} e_{Q}-\varepsilon \Gamma_{Q} u_{D}$

Consequently,

$$
\begin{aligned}
\tilde{e}_{y} & \equiv y_{p}-\hat{y}_{Q}=C x+C_{Q} x_{Q}-\left[C_{Q} x_{Q}+C_{Q} e_{Q}\right] \\
& =C x-C_{Q} e_{Q}
\end{aligned}
$$

As in [7]-[8], we define the Ideal Trajectories, but only for the ASPR Subsystem:

$\left\{\begin{array}{l}\dot{x}_{*}=A x_{*}+B u_{*}+\Gamma u_{D} \\ y_{*}=C x_{*}=0\end{array}\right.$

with $\left\{\begin{array}{l}x_{*}=S_{1}^{*} z_{D} \\ u_{*}=S_{2}^{*} z_{D}\end{array}\right.$

This is equivalent to the Matching Conditions:

$$
\left\{\begin{array}{l}
S_{1}^{*} F=A S_{1}^{*}+B S_{2}^{*}+\Gamma \theta \\
C S_{1}^{*}=0
\end{array}\right.
$$

which are known to be uniquely solvable when $C B$ is nonsingular. Since we are assuming $C B$ is nonsingular, there exists a solution to the Matching Conditions, but we do not need to know the actual solutions, since they do not appear in the final formulation of the adaptive control law.

$$
\begin{aligned}
& \text { Let }\left\{\begin{array}{l}
\Delta x \equiv x-x_{*} \\
\Delta u \equiv u_{p}-u_{*} \\
\Delta \tilde{y} \equiv \tilde{e}_{y}=C x-C_{Q} e_{Q}
\end{array}\right. \text {. Then we have } \\
& \left\{\begin{array}{l}
\Delta \dot{x}=A \Delta x+B \Delta u \\
\Delta \tilde{y}=C x-y_{*}-C_{Q} e_{Q}=C \Delta x-C_{Q} e_{Q}
\end{array}\right.
\end{aligned}
$$

because, from (14), $y_{*}=0$. This system can be rewritten:

$$
\left\{\begin{aligned}
{\left[\begin{array}{c}
\Delta \dot{x} \\
\dot{e}_{Q}
\end{array}\right] } & =\left[\begin{array}{cc}
A & 0 \\
0 & A_{Q}
\end{array}\right]\left[\begin{array}{l}
\Delta x \\
e_{Q}
\end{array}\right]+\left[\begin{array}{l}
B \\
0
\end{array}\right] \Delta u+\varepsilon\left[\begin{array}{c}
0 \\
-\Gamma_{Q}
\end{array}\right] u_{D} \\
& =\bar{A}\left[\begin{array}{l}
\Delta x \\
e_{Q}
\end{array}\right]+\bar{B} \Delta u+\varepsilon \bar{\Gamma}_{Q} u_{D} \\
\Delta \tilde{y} & =\left[\begin{array}{ll}
C & -C_{Q}
\end{array}\right]\left[\begin{array}{l}
\Delta x \\
e_{Q}
\end{array}\right]=\bar{C}\left[\begin{array}{l}
\Delta x \\
e_{Q}
\end{array}\right]
\end{aligned}\right.
$$

Now we have the following:

Lemma: $\left(\bar{A}=\left[\begin{array}{cc}A & 0 \\ 0 & A_{Q}\end{array}\right], \bar{B}=\left[\begin{array}{l}B \\ 0\end{array}\right], \bar{C}=\left[\begin{array}{ll}C & -C_{Q}\end{array}\right]\right)$ ASPR if and only if $(A, B, C)$ ASPR.

Proof: $\bar{C} \bar{B}=\left[\begin{array}{ll}C & -C_{Q}\end{array}\right]\left[\begin{array}{l}B \\ 0\end{array}\right]=C B>0$ and

$$
\begin{aligned}
\bar{P}(s) & \equiv \bar{C}(s I-\bar{A})^{-1} \bar{B} \\
& =\left[\begin{array}{ll}
C & -C_{Q}
\end{array}\right]\left[\begin{array}{cc}
(s I-A)^{-1} & 0 \\
0 & \left(s I-A_{Q}\right)^{-1}
\end{array}\right]\left[\begin{array}{l}
B \\
0
\end{array}\right] \\
& =C(s I-A)^{-1} B=P(s)
\end{aligned}
$$

is minimum phase.\#

So there exists $G_{e}^{*}$ such that $\left(\bar{A}_{C} \equiv \bar{A}+\bar{B} G_{e}^{*} \bar{C}, \bar{B}, \bar{C}\right)$ is Strictly Positive Real (SPR) when $(A, B, C)$ is ASPR. Consequently, as is well known from the Kalman-Yacubovic Theorem:

$\exists \bar{P}, \bar{Q}>0$ э $\left\{\begin{array}{l}\bar{A}_{C}^{T} \bar{P}+\bar{P} \bar{A}_{C}=-\bar{Q} \\ \bar{P} \bar{B}=\bar{C}^{T}\end{array}\right.$

We now augment the adaptive control law with an RMF:

$\left\{\begin{array}{l}u_{p} \equiv G_{e} \tilde{e}_{y}+G_{D} \phi_{D} \\ \tilde{e}_{y} \equiv y_{p}-\hat{y}_{Q} \\ \hat{\hat{x}}_{Q}=A_{Q} \hat{x}_{Q}+B_{Q} u_{p} \\ \hat{y}_{Q}=C_{Q} \hat{x}_{Q}\end{array}\right.$

with modified adaptive gains:

$$
\left\{\begin{array}{l}
\dot{G}_{e}=-\tilde{e}_{y} \tilde{e}_{y}^{T} h_{e} ; h_{e}>0 \\
\dot{G}_{D}=-\tilde{e}_{y} \phi_{D}^{T} h_{D} ; h_{D}>0
\end{array}\right.
$$

Finally, we have the following stability result:

Theorem: In (9), let $(A, B, C)$ ASPR, $A_{Q}$ stable, $\phi_{D}$ bounded. Then the Augmented Adaptive Controller with RMF in (19)-(20) produces $e_{y}=y_{p}$ and $e_{Q}$ ultimately bounded into a ball of radius $R_{*} \equiv \varepsilon \frac{\left(1+\sqrt{p_{\text {max }}}\right)}{a \sqrt{p_{\text {min }}}} M_{v}$ with exponential rate and bounded adaptive gains $\left(G_{e}, G_{D}\right)$. Proof: From (19), $u_{p} \equiv G_{e} \tilde{e}_{y}+G_{D} \phi_{D} \Rightarrow$ 


$$
\begin{aligned}
& \Delta u \equiv u_{p}-u_{*} \\
& =\left[G_{e} \tilde{e}_{y}+G_{D} \phi_{D}\right]-\left[S_{2}^{*} L\right] \phi_{D} \\
& =G_{e}^{*} \tilde{e}_{y}+\Delta G \eta \\
& \text { where }\left\{\begin{array}{l}
\Delta G_{e} \equiv G_{e}-G_{e}^{*} \\
\Delta G_{D} \equiv G_{D}-\left(S_{2}^{*} L\right) \\
\Delta G \equiv G-G_{*}=\left[\begin{array}{ll}
\Delta G_{e} & \Delta G_{D}
\end{array}\right] . \text { Then } \\
\eta \equiv\left[\begin{array}{l}
\tilde{e}_{y} \\
\phi_{D}
\end{array}\right]
\end{array}\right. \\
& \left\{\begin{array}{l}
\dot{\zeta}=\bar{A} \zeta+\bar{B} \Delta u=\bar{A}_{C} \zeta+\bar{B} w+\varepsilon \bar{\Gamma}_{Q} u_{D} \\
\tilde{e}_{y}=\bar{C} \zeta
\end{array}\right. \\
& \text { with } \zeta \equiv\left[\begin{array}{l}
\Delta x \\
e_{Q}
\end{array}\right], w \equiv \Delta G \eta, \bar{A}_{C} \equiv \bar{A}+\bar{B} G_{e}^{*} \bar{C}
\end{aligned}
$$

From (20), we can see that

$$
\dot{G}=\Delta \dot{G}=-\tilde{e}_{y} \eta^{T} h ; h \equiv\left[\begin{array}{cc}
h_{e} & 0 \\
0 & h_{D}
\end{array}\right]>0
$$

Since $(A, B, C)$ is ASPR, and by the lemma, so is $(\bar{A}, \bar{B}, \bar{C})$, we can we can use the following result from [20] where $v \equiv \bar{\Gamma}_{Q} u_{D}$ is bounded because the disturbance $u_{D}=L \phi_{D}$ is bounded:

Result: Consider the nonlinear, coupled system of differential equations,

$$
\left\{\begin{array}{l}
\dot{\zeta}=\bar{A}_{c} \varsigma+\bar{B}\left(G(t)-G^{*}\right) \eta+\varepsilon v \\
\tilde{e}_{y}=\bar{C} \varsigma \\
\dot{G}(t)=-\tilde{e}_{y} \eta^{\top} h-a G(t)
\end{array}\right.
$$

where $G^{*}$ is any constant matrix and $h$ is any positive definite constant matrix, each of appropriate dimension. Assume the following:

i) the triple $(\bar{A}, \bar{B}, \bar{C})$ is $S P R$,

ii) $\exists M_{K}>0 \ni\left\|\left(G^{*}\right)^{T} G^{*}\right\| \leq M_{K}$, using the trace norm,

iii) $\exists M_{v}>0$ э $\sup _{t \geq 0}\|v(t)\| \leq M_{v}$,

iv) $\exists a>0 \quad \ni a \leq \frac{q_{\min }}{2 p_{\max }}$, and

v) $h$ satisfies $\left\|h^{-1}\right\|_{2} \leq\left(\frac{\varepsilon M_{v}}{a M_{K}}\right)^{2}$, where $p_{\min }$, $p_{\max }$ are the minimum and maximum eigenvalues of $\bar{P}$ and $q_{\min }$ is the minimum eigenvalue of $\bar{Q}$ in $\left\{\begin{array}{l}\bar{A}_{C}^{T} \bar{P}+\bar{P} \bar{A}_{C}=-\bar{Q} \\ \bar{P} \bar{B}=\bar{C}^{T}\end{array}\right.$

Then the matrix $G(t)$ is bounded and the state $\zeta(t)$ exponentially approaches the ball of radius
$R_{*} \equiv \varepsilon \frac{\left(1+\sqrt{p_{\max }}\right)}{a \sqrt{p_{\min }}} M_{v}$ with $\varepsilon>0$.

From this result, we have $\zeta$ is ultimately bounded into the ball of radius $R_{\star}$, which leads to $e_{y} \equiv y_{p}=y_{p}-y_{*}=C \Delta$ and $e_{Q}$ ultimately bounded as well. Therefore $G=G_{*}+\Delta G$ is bounded, as desired. \#

Consequently, the radius of the error ball $R_{*} \equiv \varepsilon \frac{\left(1+\sqrt{p_{\max }}\right)}{a \sqrt{p_{\text {min }}}} M_{v}$ is determined by the size of $\varepsilon$, which is related to the amount of disturbance leakage into the $\mathrm{Q}$ modes, and the desired rate of convergence, $a$.

\section{Simulation Results With RMF}

A residual mode filter was designed for the drivetrain torsional mode of the FAST simulation of the CART. The RMF was added to the baseline PI pitch controller for Region 3 operation in the presence of the drivetrain torsional mode and the first flapwise blade mode. The simulation was run with the following DOFs enabled: generator, drivetrain rotational-flexibility mode, and first flapwise blade mode. The results of the RMF augmented baseline PI controller for Region 3 with step wind inflow can be seen in fig. 4(a). The adaptive controller with rejection of step disturbances as described in section III was augmented with the same RMF. In both cases, the controller gains remained as they were prior to adding the RMF. Results of the augmented adaptive controller operating with step wind inflow and the drivetrain torsional mode DOF and the first flapwise blade mode DOF enabled can be seen in fig. 4(b). In both cases, the addition of the RMF improved the generator speed regulation of the controllers.

\section{CONCLUSIONS}

We proposed an adaptive controller augmented with a residual mode filter. The RMF is used to accommodate troublesome modes in the system that would otherwise interfere with the almost strict positive real requirement for the adaptive controller. The new theory presented in this paper accounts for leakage of the disturbance term into the $\mathrm{Q}$ modes. We used this new approach to design an adaptive collective pitch controller for Region 3 operation of a wind turbine. The controller was able to improve generator speed regulation in a high-fidelity simulation of a wind turbine with structural modes. The efficient operation of large utility scale wind turbines could be improved with this new control approach. Additionally, other flexible structure control problems, where feedback in the control loop causes instabilities and a more precise method of filtering the feedback signal is desired, could benefit from adaptive control augmented with a RMF.

\section{REFERENCES}

[1] Fingersh, LJ, Johnson, KE. Baseline results and future plans for the NREL Controls Advance Research Turbine. Proceedings of the $23^{\text {rd }}$ 
AIAA Aerospace Sciences Meeting and Exhibit Wind Energy Symposium 2004; 87-93.

[2] Wright, AD, Stol, KA, Fingersh, LJ. Progress in implementing and testing state-space controls for the controls advanced research turbine. Proceedings of the $24^{\text {th }}$ AIAA Aerospace Sciences Meeting and Exhibit Wind Energy Symposium 2005; 88-100.

[3] Narendra, KS, Annaswamy AM. Stable Adaptive Systems. PrenticeHall: New Jersey, 1989.

[4] Åström, KJ, Wittenmark, B. Adaptive Control ( $2^{\text {nd }}$ edition). AddisonWesley: Massachusetts, 1995.

[5] Wen, JT, Balas, MJ. Robust adaptive control in Hilbert space. Journal of Mathematical Analysis and Application 1989; 143(1): 1-26.

[6] Balas, MJ. Finite-dimensional direct adaptive control for discrete-time infinite-dimensional linear systems. Journal of Mathematical Analysis and Applications 1995; 196(1): 153-171.

[7] Fuentes, RJ, Balas, MJ. Direct adaptive rejection of persistent disturbances. Journal of Mathematical Analysis and Applications 2000; 251(1): 28-39.

[8] Frost, SA, Balas, MJ, and Wright, AD. Direct adaptive control of a utility-scale wind turbine for speed regulation, International Journal of Robust and Nonlinear Control, 2009, 19(1): 59-71, DOI: $10.1002 /$ rnc. 1329

[9] Freeman, JB, Balas, MJ. Direct model-reference adaptive control of variable speed horizontal-axis wind turbines. Wind Engineering 1998; 22: $209-218$

[10] Johnson, KE, Fingersh, LJ. Adaptive pitch control of variable-speed wind turbines. Collection of Technical Papers $-45^{\text {th }}$ AIAA Aerospace Sciences Meeting and Exhibit 2007; 18:12391-12398.

[11] Jonkman, JM, Buhl, ML. FAST user's guide, National Renewable Energy Laboratory, NREL/EL-500-38230, Golden, Colorado, August, 2005.
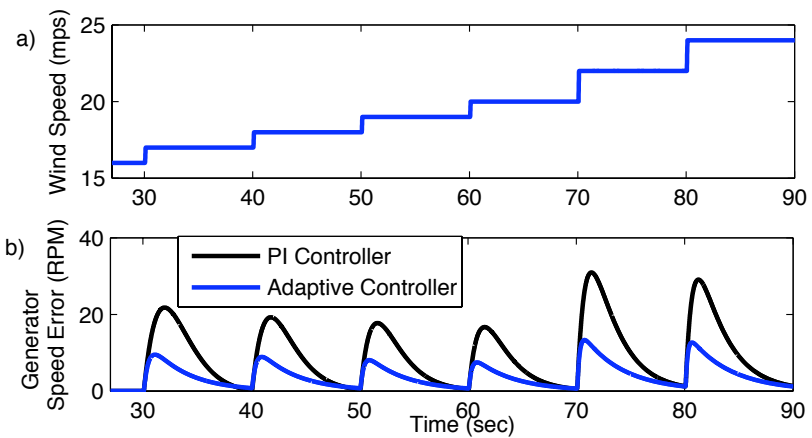

Fig. 1. (a) Step wind inflow used in simulations, (b) generator speed errors for simulation with drivetrain torsional mode and first flapwise blade mode DOFs disabled.

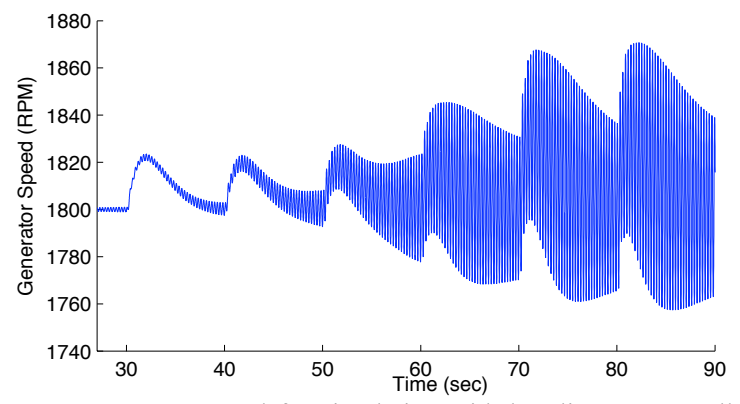

Fig. 2. Generator speed for simulation with baseline PI controller and drivetrain torsional and first flapwise blade mode DOFs enabled.
[12] Wright, AD, Balas, MJ, Fingersh, LJ. Testing state-space controls for the controls advanced research turbine. Transactions of the ASME. Journal of Solar Energy Engineering 2006; 128(4): 506-515.

[13] Laino, DJ, Hansen, AC. User's guide to the computer software routines AeroDyn interface for ADAMS ${ }^{\circledR}$. Salt Lake City, Utah Windward Engineering, LC, September 2001,

[14] Johnson, C.D. Theory of disturbance-accommodating controllers. Control \& Dynamic Systems, Advances in Theory and Applications, Leondes, CT. ed. Academic Press: New York, 1976; 12: 387-489.

[15] Frost, SA, Balas, MJ, and Wright, AD. Adaptive Control of a UtilityScale Wind Turbine Operating in Region 3, Proceedings of the $28^{\text {th }}$ AIAA Aerospace Sciences Meeting and Exhibit Wind Energy Symposium, Orlando 2009

[16] National Renewable Energy Lab., NWTC Design Codes (FAST), $\mathrm{http} / / /$ wind.nrel.gov/designcodes/simulators/fast/, National Renewable Energy Lab., Golden, CO, May 26, 2008.

[17] Wright, AD, Balas, MJ. Design of controls to attenuate loads in the controls advanced research turbine. Transactions of the ASME. Journal of Solar Energy Engineering 2004; 126(4): 1083-91.

[18] Balas, MJ, Finite-dimensional controllers for linear distributed parameter systems: Exponential stability using Residual Mode Filters," J. Mathematical Analysis \& Applications, Vol. 133, pp. 283296, 1988

[19] Frost, S. A., Balas, M. J., and Wright, A. D. "Modified adaptive control for region 3 operation in the presence of wind turbine structural modes", ASME Wind Energy Symposium, Orlando, 2010.

[20] Fuentes, R J and Balas, M J, "Robust Model Reference Adaptive Control with Disturbance Rejection", Proceedings of the American Control Conference, 2002.

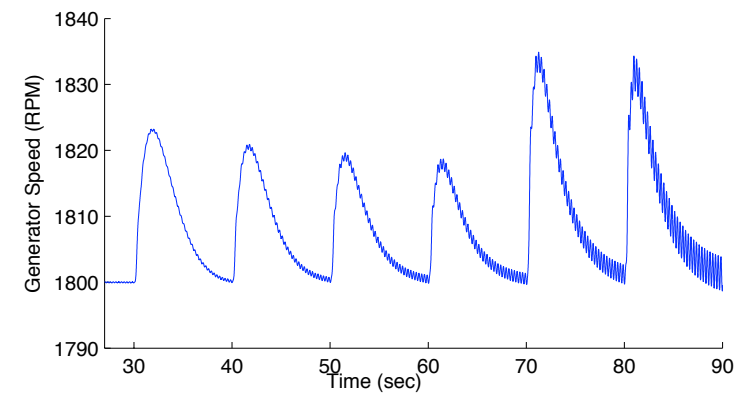

Fig. 3. Generator speed for simulation with drivetrain torsional and first flapwise blade DOFs enabled for baseline PI controller with low-pass filter on generator speed.
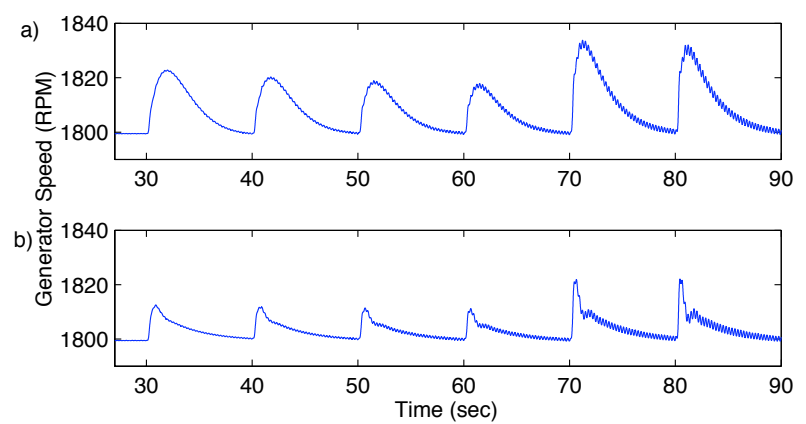

Fig. 4. Generator speed for (a) baseline PI controller with RMF and (b) adaptive controller with RMF. Simulation had drivetrain torsional mode and first flapwise blade mode DOFs enabled. RMF was designed for drivetrain torsional mode. 